\title{
Texture investigation of zone-melted NBCO superconductor using EBSD technique
}

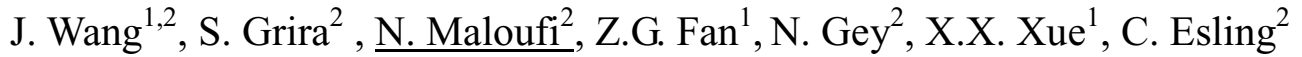 \\ 1. School of Material and Metallurgy, Northeastern University, Shenyang 110004, China \\ 2. LETAM UMR - CNRS 7078, University Paul Verlaine - Metz - Ile du Saulcy - 57012 Metz \\ France \\ nabila.maloufi@univ-metz.fr
}

\begin{abstract}
:
NBCO bars were textured using zone melting method under low oxygen partial pressure. By means of electron backscattered diffraction (EBSD) method, quantitative texture analysis of zone-melted NBCO samples was studied. High-quality Kikuchi patterns were obtained, allowing the automated mapping on samples. The EBSD results are presented in the form of local orientation maps, phase maps and pole figures, revealing the crystal growth characteristics of the zone melted sample and the distribution of misorientations for the NBCO phase.
\end{abstract}

\section{Introduction}

Compared to $\mathrm{YBCO}, \mathrm{NdBa}_{2} \mathrm{Cu}_{3} \mathrm{O}_{7-\delta}(\mathrm{NBCO})$ has better superconducting properties under high magnetic field [1]. Optimal performances can only be achieved in such NBCO samples with a well-textured microstructure. The partial zone-melting technique seems to be one of the most appropriate methods for obtaining textured NBCO samples [2]. For Nd-Ba-Cu-O system, zone-melting process takes place by moving a sample through a narrow hot zone with a thermal gradient. This process leads to the decomposition into solid phase $\mathrm{Nd}_{4} \mathrm{Ba}_{2} \mathrm{Cu}_{2} \mathrm{O}_{10}$ $(\mathrm{Nd} 422)$ and liquid phase $\mathrm{BaCuO}_{2}$ and $\mathrm{CuO}$, which recrystallize by peritectic reaction and grow with preferential orientation. Moreover, low oxygen partial pressure technology has routinely been employed for making NBCO superconductors [3-5]. The advantage of melting texture in low oxygen partial pressure is that it can reduce the substitution of $\mathrm{Nd}$ for $\mathrm{Ba}$ in NBCO samples to improve the superconducting performances.

In this study, zone melting method under low oxygen partial pressure was used to obtain textured NBCO bars. EBSD measurements including automated mapping were performed on samples. The texture analysis of the samples is discussed.

\section{Experimental}

Zone-melted NBCO samples were prepared using the procedure described in Ref.[6]. NBCO starting powder mixed with an additional amount of $10 \mathrm{wt} \% \mathrm{Nd} 422$ was pressed into $6 \mathrm{~cm}$ long bars, then calcined. After zone-melting in low oxygen partial pressure $\left(1 \% \mathrm{O}_{2}+99 \% \mathrm{Ar}\right)$, oxygen treatment was performed on the samples. Before EBSD scan the polishing procedure of the samples was optimized because the sample surface quality largely determines whether or not Kikuchi patterns obtained from the material will be clear and can be indexed [7].

A JEOL 6500F FEG-SEM equipped with the HKL Channel5 EBSD analysis system was used. The Kikuchi patterns were generated at an acceleration voltage of $15 \mathrm{kV}$ and recorded using a Nordlys II camera. To produce a crystallographic orientation map, the electron beam was 
scanned over a selected surface area and the resulting Kikuchi patterns were automatically indexed and analyzed using analysis software package [8].

\section{Results and discussion}

The band contrast image presented in figure 1(a) is similar to a standard SEM image, but contains quality information of the recorded Kikuchi patterns. A lighter color indicated a higher recorded image quality. In this map, the presence of some stripes of polishing traces can be recognized. Figure 1(b) presents the phase map. The Nd123 phase is indicated in blue, the Nd422 phase in red. Figure 1(c) and Figure 1(d) separately present the crystallographic orientations of Nd123 phase and Nd422 phase in inverse pole figure (IPF) maps. In the socalled IPF map, the grains are shaded by their respective crystallographic orientation normal to the sample surface. This normal is associated with $\mathrm{Z}$ direction as indicated near the colorcoded stereographic triangle. The IPF map shows that the Nd123 grains are preferentially oriented but the c-axes are out of the sample normal direction. Some misindexed points in Nd123 phase can be found. This is due to the presence of defects and porosities in the sample surface, near which the Kikuchi patterns become ambiguous. Large Nd422 grains of tenmicrometer-size are formed. Small grains of Nd422 are located near the cracks within the Nd123 matrix. These Nd422 particles are randomly distributed and do not show any preferred orientation in IPF map.

Figure 2 presents for the Nd123 phase the distribution of the angle between the c-axes and the macroscopic sample normal direction. Two peaks can be found in the graph with rainbow scale. The position of the peak represents the mean orientation of the Nd123 grains with respect to the normal direction, the width of the peak represents the spreading of the orientation distribution. There is a main peak with grains having the c-axis tilted at $43^{\circ}$ to the sample normal direction, and a secondary peak tilted at $55^{\circ}$. According to the width of large peak, most misorientations between $\mathrm{Nd} 123$ grains are below $6^{\circ}$, while in left part of the map, corresponding to the small peak, one subdomain with misorientation up to $10^{\circ}$ should be noticed. Some thermal instabilities can be responsible for such subdomains.

Such results can also be deduced more precisely from the EBSD pole figure in Figure 3(a). This means that during zone-melting process, the a-b planes generally did not grow along the sample moving direction but at approximately an angle of $45^{\circ}$ to this direction. A top view diagram describing the orientation geometry of Nd123 unit cell in zone-melted sample is given in Figure 3(b). Similar characteristics of crystal growth in YBCO zone-melted samples have been explained by the consequence of the selected grain growth mechanism [9-11]. These results can also be applied to NBCO. 

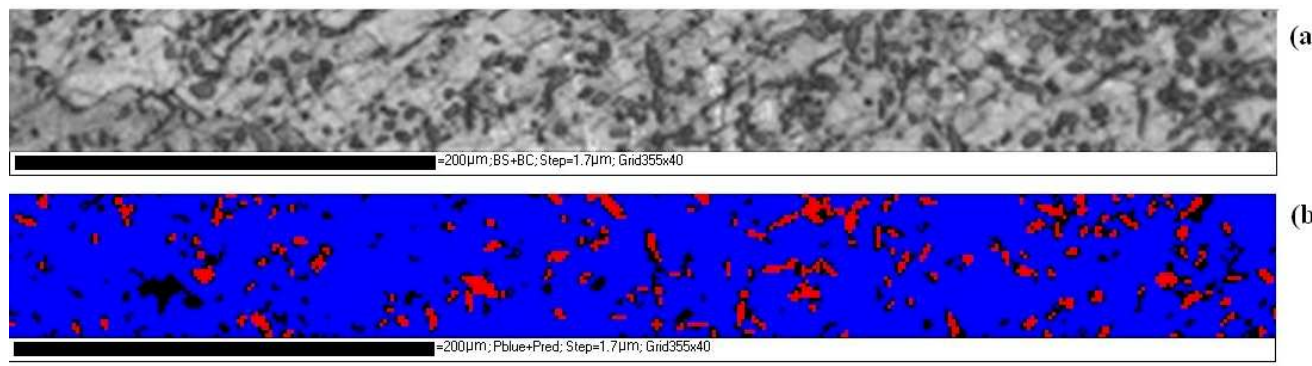

(b)
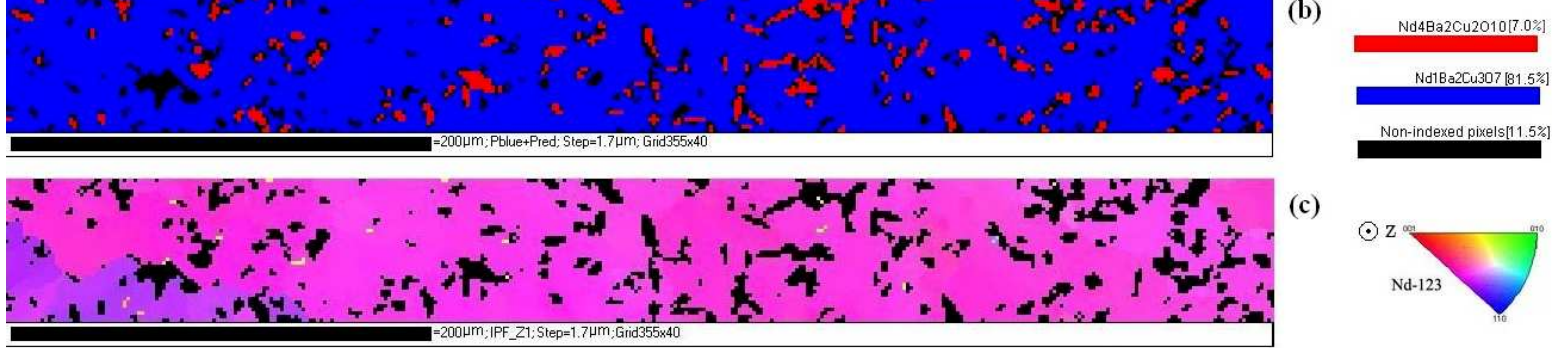

(c)
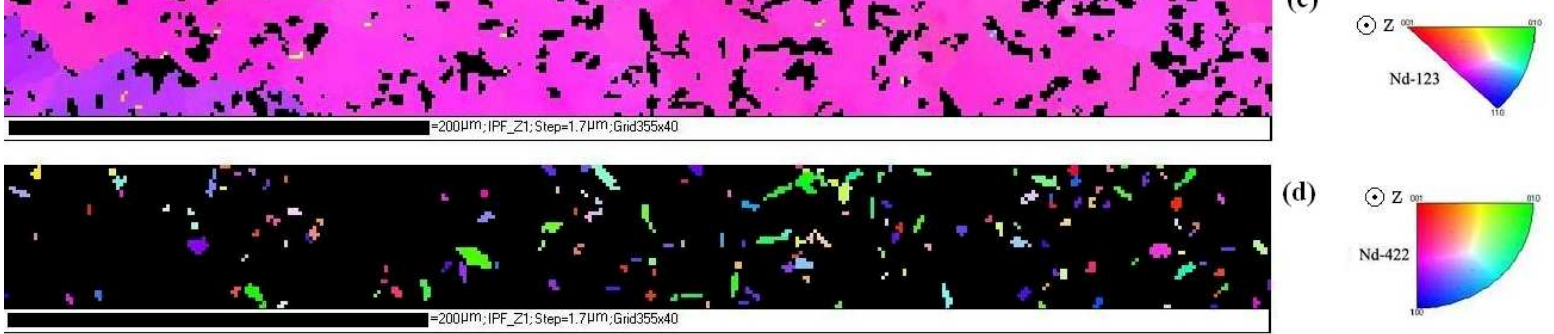

(d)

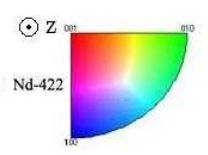

Fig. 1. EBSD mappings of zone-melted sample. (a) is a band contrast image, which is similar to a standard SEM image. (b) is the phase map: The Nd123 phase is indicated in blue, the Nd422 phase in red and the non indexed pixels [11.5\%] are in black. Composition and percentage are given above the color bars: red ( $\mathrm{Nd} 4 \mathrm{Ba} 2 \mathrm{Cu} 2 \mathrm{O} 10$ [7.0\%] ), blue ( $\mathrm{Nd} 1 \mathrm{Ba} 2 \mathrm{Cu} 3 \mathrm{O} 7$ [81.5\%] ). (c) for $\mathrm{Nd} 123$ and (d) for $\mathrm{Nd} 422$ are inverse pole figure (IPF) maps for each phase separately, while the other phase is indicated as black. The cristallographic orientations can be obtained from the color-coded stereographic triangles in which $\mathrm{Z}$ direction corresponds to the surface normal [001].

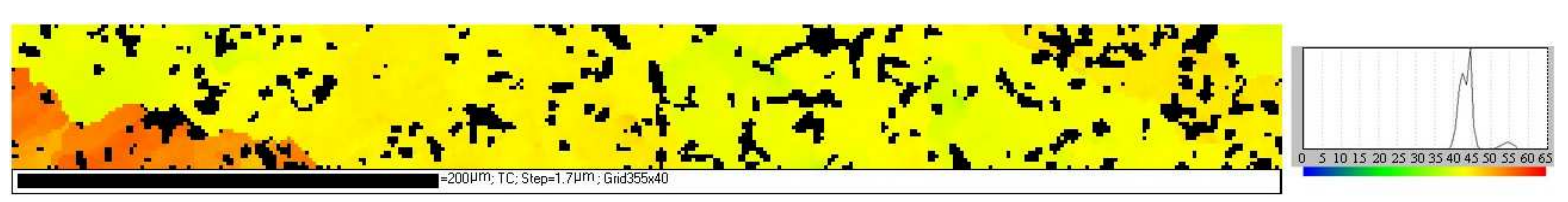

Fig. 2. Orientation map representing the orientation of the c-axes with respect to the macroscopic sample normal direction.

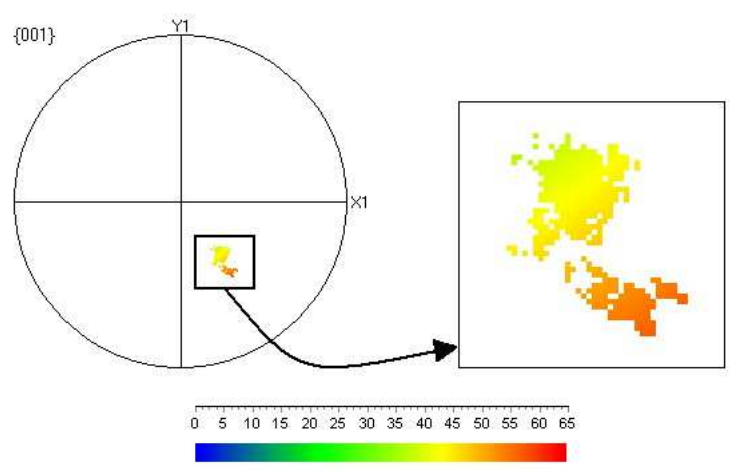

(a)

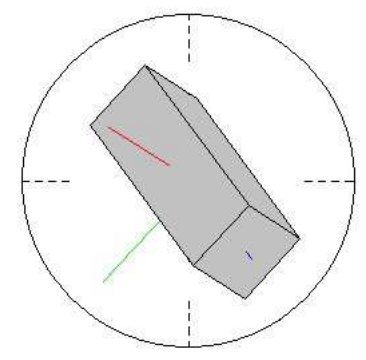

(b)

Fig. 3. (a) EBSD pole figure of the (001) plane. (b)Geometry of Nd123 unit cell in zone-melted sample

\section{Conclusion}

NBCO textured bars have been prepared by zone-melting method in low oxygen partial pressure. The distribution of orientations in zone-melted samples has been analysed by EBSD method. The $\mathrm{a}-\mathrm{b}$ planes in zone-melted superconductor samples generally were not aligned 
with the sample moving direction but orientated at approximately an angle of $45^{\circ}$ due to the selected grain growth mechanism. It was found that it is also the case for the Nd123 phase. It oriented preferentially with c-axis at $43^{\circ}$ from the bar axis. Some subdomains can be found in the misorientation map. Such subdomains are mainly caused by thermal instabilities. Nd422 particles, which can act as good pinning centers, show homogeneous distribution and nearly random orientations.

\section{References}

1. M. Murakami, N. Sakai, T. Higuchi, S. I. Yoo, Melt-processed light rare earth element- Ba-Cu-O[J], Supercond. Sci. Technol., 9:1015-1032 (1996).

2. R. Cabré, J. Gavaldà, J. Massons, R. Solé, X. Ruiz, M. Aguiló and F. Diaz, Improvements in obtaining YBCO single domains using the partial melting zone method [J], Supercond. Sci. Technol., 11:30-34 (1998).

3. P. Diko, H. Kojo and M. Murakami, Microstructure of $\mathrm{Nd}-\mathrm{Ba}-\mathrm{Cu}-\mathrm{O}$ superconductors prepared by oxygencontrolled-melt-growth method[J], Physica C, 276(3-4):185-196 (1997).

4. H. Wu, M. J. Kramer, K. W. Dennis, and R. W. McCallum, Mechanism for flux pinning in $\mathrm{NdBa}_{2} \mathrm{Cu}_{3} \mathrm{O}_{7-\delta}$ melttextured in low oxygen partial pressure[J], Appl. Phys. Lett., 71 (24):3572-3574 (1997).

5. I. Monot, F. Tancreta, P. Laffez, G. Van Tendeloo, G. Desgardin, Microstructure and properties of oxygen controlled melt textured $\mathrm{NdBaCuO}$ superconductive ceramics[J], Materials Science and Engineering B, 65(1):26-34 (1999).

6. Z. G. Fan, W. Y. Gao, F. H. Li, J. Wang, D. H. Soh, Preparation of NdBaCuO superconductor by zone-melting under low oxygen partial pressure[J], Physica C, 386:241-244 (2003).

7. V. A. Koblischka, M. R. Koblischka, P. Simon, F. Mücklich, M. Murakami, Electron backscatter diffraction study of polycrystalline $\mathrm{YBa}_{2} \mathrm{Cu}_{3} \mathrm{O}_{7-\delta}$ ceramics [J], Physica C, 382:311-322 (2002).

8. J. Wang, N. Maloufi, N. Gey, C. Esling, Magnetic melt process and texture analysis of high temperature superconductor $\mathrm{YBa}_{2} \mathrm{Cu}_{3} \mathrm{O}_{7-\delta}$ for electrical applications, Ferroelectrics, 353:249-257 (2007).

9. S. Marinel, J. Provost, G. Desgardin, The crystal growth anisotropy of $\mathrm{YBa}_{2} \mathrm{Cu}_{3} \mathrm{O}_{7-\delta}$ fabricated by the MTG method in a microwave cavity [J], Physica C, 294:129-139 (1998).

10. Y. Nakamura, K. Furuya, T. Izumi, Y. Shiohara, Crystal orientation and growth anisotropy of $\mathrm{YBa}_{2} \mathrm{Cu}_{3} \mathrm{O}_{6+\delta}$ fabricated by directional solidification method $[\mathrm{J}]$, J. Mater. Res., 9(6):1350-1356 (1994).

11. Y. Imagawa, Y. Shiohara, Orientation control of Pt added $\mathrm{YBa}_{2} \mathrm{Cu}_{3} \mathrm{O}{ }_{6+x}$ by the directional solidification method[J], Physica C, 262:243-248 (1996). 